\title{
Xyloglucan, hibiscus and propolis to reduce symptoms and antibiotics use in recurrent UTIs: a prospective study
}

\author{
Tommaso Cai ${ }^{*, 1}$, Irene Tamanini ${ }^{1}$, Andrea Cocci ${ }^{2}$, Fabrizio di Maida ${ }^{2}$, Patrizio Caciagli ${ }^{3}$, \\ Serena Migno ${ }^{4}$, Liliana Mereu ${ }^{4}$, Saverio Tateo ${ }^{4}$, Gianni Malossini' ${ }^{1}$, Alessandro Palmieri ${ }^{5}$, \\ Paolo Verze ${ }^{5}$, Vincenzo Mirone ${ }^{5} \&$ Truls E Bjerklund Johansen ${ }^{6}$ \\ ${ }^{1}$ Department of Urology, Santa Chiara Hospital, Trento, Italy \\ ${ }^{2}$ Department of Urology, University of Florence, Florence, Italy \\ ${ }^{3}$ Department of Laboratory Medicine, Santa Chiara Regional Hospital, Trento, Italy \\ ${ }^{4}$ Department of Gynaecology \& Obstetrics, Santa Chiara Regional Hospital, Trento, Italy \\ ${ }^{5}$ Department of Urology, University of Naples, Federico II, Naples, Italy \\ ${ }^{6}$ Department of Urology, Oslo University Hospital, Oslo, Norway \& Institute of Clinical Medicine, University of Oslo, Norway \\ *Author for correspondence: Tel.: +39 0461 903306; Cell: +39 333986 4943; Fax: +39 0461 903101; ktommy@libero.it
}

\begin{abstract}
Aim: To evaluate the efficacy of a medical device containing xyloglucan, hibiscus and propolis in the management of recurrent urinary tract infections (rUTIs). Patients \& methods: Sixty-one women affected by rUTIs received this medical device, one capsule a day for 15 days (one cycle every month, for 6 months), in an observational, prospective study. Clinical and microbiological evaluations were performed at baseline and 1, 3 and 6 months from enrolment. Results: At first follow-up, 41 reported a clinical improvement and a return to their clinical status before UTI, while 47 and 51 did so at the second and third follow-up evaluations. A statistically significant clinical improvement was reported at each follow-up visit (quality of life [QoL] 94.2 vs 98.6; QoL 94.1 vs 98.7; QoL 94.2 vs 99.1; p < 0.001). A statistically significant reduction in antibiotic use was reported. Conclusion: This medical device is able to improve quality of life in women with rUTIs, reduce recurrences and antibiotic use.
\end{abstract}

Keywords: antibiotic stewardship • ibiscus • propolis • quality of life • urinary tract infections $\bullet$ xyloglucan

Urinary tract infections (UTI) are one of the most common infections in outpatients in the USA with high costs and worse patients' quality of life ${ }_{[1]}$. The prevalence of UTIs in everyday clinical outpatients ranges from 1 to $6 \%$ of all medical visits. Recurrent UTI refers to $\geq 2$ infections in 6 months or $\geq 3$ infections in 1 year ${ }_{[1]}$. Recurrent uncomplicated UTIs are common among young, healthy women even though they generally have anatomically and physiologically normal urinary tracts. Moreover, after an initial UTI, approximately 20-30\% of women with a UTI will have a second UTI within 6 months, and 3\% will experience a third UTI during that time period ${ }_{[2]}$. Several authors found that frequent intercourse, vulvovaginal atrophy, change of the local bacterial flora, history of UTIs during premenopause or in childhood, treatment of asymptomatic bacteriuria, family history and a nonsecretor blood type are risk factors for recurrent UTIs [2]. The management of recurrent uncomplicated urinary tract infections (rUTIs) in women is still the subject of great discussion. On the one hand, we need effective treatment for symptom relief and to reduce the risk of acute episode recurrence ${ }_{[3]}$. On the other, an antibiotic-sparing approach, due to the worldwide emergence of antibiotic resistance, is urgently required ${ }_{[3,4]}$. In this regard, nonantibiotic oral supplements, including phytotherapy and nutraceuticals, seem to be an attractive alternative to antibiotic therapy to ameliorate symptoms related to UTIs and lower the rate of symptomatic recurrence. Moreover, according to the guidelines of the European Association of Urology (EAU), all clinicians should recommend counselling, behavioral modification and nonantimicrobial measures $[5,6]$. On the basis of these considerations, here we focused our attention on a medical device containing xyloglucan, hibiscus and propolis in the management of women affected by rUTIs. Xyloglucan acts mechanically into the bowel lumen for 


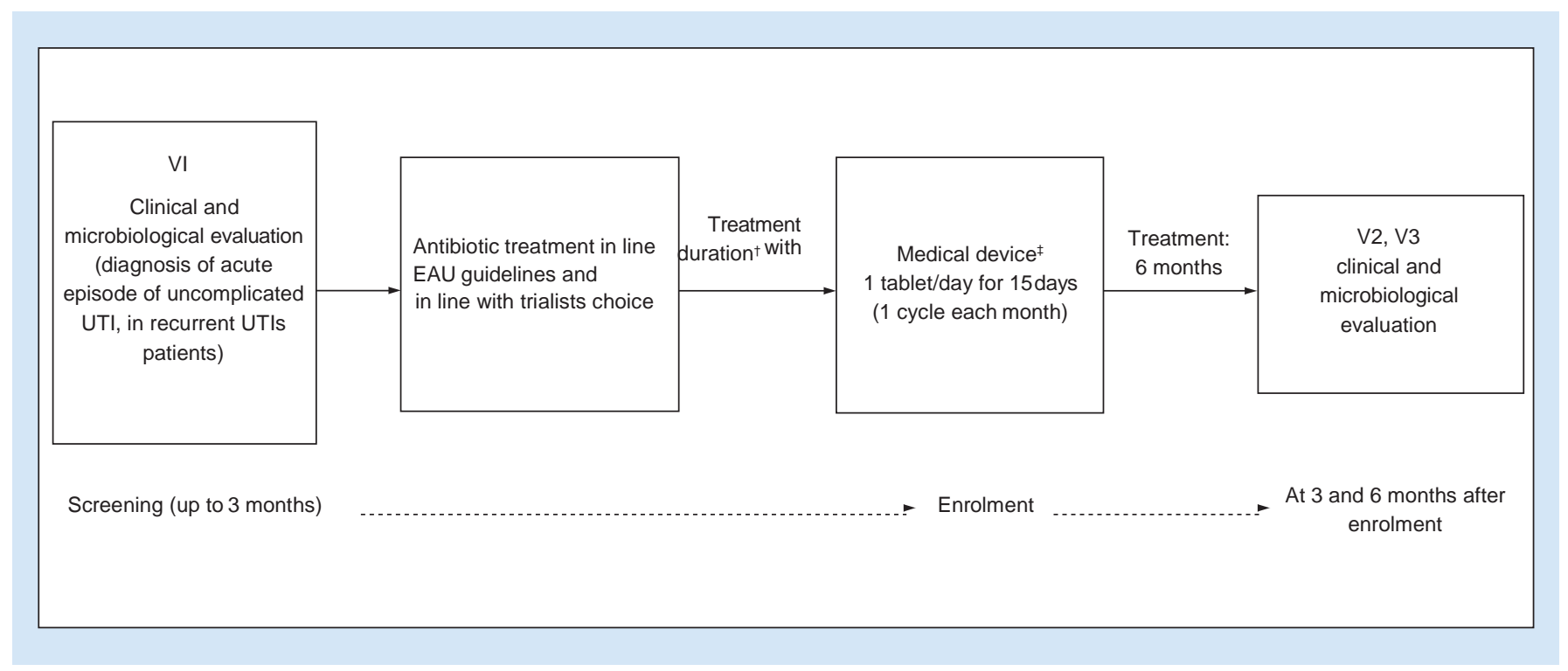

Figure 1. The figure shows the study schedule.

tTreatment duration in line with the antibiotic used.

¥Medical device containing xyloglucan, hibiscus and propolis.

EAU: European Association of Urology; UTI: Urinary tract infections.

preventing the adhesion of uropathogens with the intestinal mucosa, which is the first step in their proliferation and subsequent passage to the urinary tract $[7,8]$. This medical device is also able to control bacterial growth through the acidification of urine $\mathrm{pH}$ by hibiscus and propolis and to reduce local urinary symptoms related to bladder mucosa inflammation $[9,10]$. Two clinical trials demonstrated a clinical role of this device in the management of patients affected by UTIs $[7,11]$. This consideration led us to conduct a prospective observational study in order to evaluate the efficacy of a medical device containing xyloglucan, hibiscus and propolis in the prophylaxis of symptomatic episodes in women affected by rUTIs focusing on both capability to reduce the number of symptomatic recurrences and efficacy in improving (QoL).

\section{Materials \& methods}

\section{Study design \& treatment schedule}

From January to September 2018, in a single center observational, prospective study, women with rUTIs who attended the center, were enrolled. All women were required to sign a dedicated written informed consent before proceeding with enrollment. All women with rUTIs, defined as $\geq 2$ infections in 6 months or $\geq 3$ infections in 1 year, were considered for this study. We excluded all women with the following conditions: evidence of overactive bladder; anatomical abnormalities or previous surgery of the urinary tract; complicated UTI; intake of antibiotics 4 weeks before enrollment; pregnancy; urinary catheterization (both indwelling and intermittent). Moreover, we excluded all patients with active infection by sexually transmitted pathogens. Immediately before enrolment a clinical and microbiological evaluation was performed by administering QoL questionnaires and performing a preliminary midstream urine culture, in line with Cai et al. [3,12]. All screened women were informed about the nature of the study and about the fact that the suggested product is already present in Italy. Immediately before enrolment all patients were treated with antibiotics in line with EAU guidelines on urological infections in order to obtain infection free status at baseline [6]. All enrolled patients underwent the following treatment schedule, after antibiotics: one capsule a day for 15 days (one cycle every month, for 6 months). Clinical and microbiological analyses were carried out at baseline (T0) and during the follow-up at 1 month (T1), 3 (T2) and 6 months (T3) from enrolment. In case of acute symptomatic episode of UTI, all patients were treated with antibiotics in line with EAU guidelines on urological infections and according to the results of the antibiogram ${ }_{[5,6]}$. Figure 1 shows the study schedule. 


\section{Outcome measures}

The main variables were the reduction of symptomatic episodes and improvement in QoL from baseline to the different follow-up periods. Microbiological cure was also considered as an outcome measure. In order to evaluate the reduction of symptomatic episodes due to the treatment, we considered the number of recurrences in the year before enrolment and we compared them with those obtained at the different follow-up visits. Finally, the difference between the period before enrolment and the period after it, in terms of antibiotic consumption (antibiotic treatment cycle) was considered as a secondary variable. Antibiotic consumption before and after treatment was calculated by asking General Practitioners (GPs) for the number of antibiotic prescriptions for each patient. Moreover, all patients were asked about the number of antibiotics taken in the two periods. Data from patients and General Practitioners were then matched.

\section{Composition \& characterization of the extracts used}

All patients were treated in line with the manufacturers instructions (ZAMBON ITALIA - Bresso - Italy - https: //www.zambonpharma.com/it/it/com). Each administration contained a combination of the following ingredients: xyloglucan, extracted from the seeds of the tamarind tree (Tamarindus indica) and extracts of Hibiscus sabdariffa and propolis. This medical device is produced by Noventures, Barcelona, Spain. The medical device is named in Italy MONURELLE PLUS \$. Each capsule contains: xyloglucan $100 \mathrm{mg}$, gelatin $50 \mathrm{mg}$, propolis $100 \mathrm{mg}$, H. sabdariffa $100 \mathrm{mg}$. In other countries, the product is referred to as UTIPRO PLUS\$.

\section{Clinical \& microbiological considerations Enrolment time \& questionnaires}

In accordance with EAU guidelines on urological infections, the diagnosis of UTI was defined according to the following parameters: patient reported symptoms and patient interview, physical examination, bed-side dip- stick urinalysis and urine culture ${ }_{[5,6]}$. All microbiological and laboratory analyses were performed as previously described ${ }_{[13]}$. In brief, all clean-catch midstream urine samples collected at room temperature were immediately taken to the laboratory under refrigerated conditions and analyzed. All urine samples were analyzed for common bacteria and yeasts, aliquoted for DNA extraction and PCR testing for Chlamydia trachomatis, Neisseria gonorrhoeae and urogenital Mycoplasma. Microbiological culture was performed according to the procedure described by Hooton $e t$ al. [14], and all other microbiological procedures and DNA extraction were performed in line with Mazzoli et al. [12, 15$]$. For microbiological diagnosis, $\geq 10^{5} \mathrm{CFU} / \mathrm{ml}$ was considered the cut-off for significant pathogenic growth ${ }_{[16]}$; the plate count method was performed in line with Mazzoli et al. [12]. At the enrolment phase, all women were asked to report the number of symptomatic recurrences had in the year before and the antibiotics taken (name, dosage and course). The impact of rUTI on patients' QoL was evaluated by using an Italian version of the Quality of Well-Being, a validated, multiattribute health scale ${ }_{[17]}$. This scale was selected because it has been successfully applied to acute illnesses, whereas other quality of life scales, including the Short Form-36 (SF-36) Health Survey, are more suitable in chronic cases. Higher scores on the QoL scale reflect a higher quality of life (maximum score 100) ${ }_{[18]}$. Patients were given patients daily cards to record their clinical symptoms and adverse events (AEs), and to report missing administrations.

\section{Follow-up evaluations}

Clinical and microbiological analyses were carried out at baseline (T0) and during follow-up at 1 month (T1), 3 (T2) and 6 months (T3) from enrolment. At each follow-up evaluation, all women underwent urologic visits with QoL questionnaires and midstream urine culture. All records about AEs and missing administrations were collected at each follow-up visit. Clinical cure was defined as symptom relief and return to pre-UTI status. Treatment failure was defined as the evidence of microbiological colonization of at least $10^{5}$ CFU per milliliter (CFUs/ml) of a single uropathogen in the midstream urine culture associated with the persistence of urinary tract symptoms. Transition from symptomatic UTI to asymptomatic bacteriuria was not considered as treatment failure. In case of treatment failure, patients underwent a short course of antibiotic therapy according to the antibiogram. However, in case of treatment failure, the patients were not excluded, but included in a subgroup analysis. At the last follow-up, all women were asked to report the number of antibiotic treatment cycles (name, dosage and course) used during the study period. The difference between the periods before and after enrolment, in terms of antibiotics consumption, was analyzed. 


\section{Table 1. Patient's sociodemographic anamnestic, clinical characteristics at enrolment time.}

\begin{tabular}{|c|c|}
\hline No. of total patients & 61 \\
\hline Median age (range) & $41.3(23-53)$ \\
\hline \multicolumn{2}{|l|}{ Educational level } \\
\hline - Primary school & - \\
\hline - Secondary school & $20(32.7)$ \\
\hline - Post-secondary education & $41(67.3)$ \\
\hline \multicolumn{2}{|l|}{ Current smoker } \\
\hline - No & $19(31.2)$ \\
\hline - Yes & $42(68.8)$ \\
\hline Sexually active (past month) & $59(96.7)$ \\
\hline \multicolumn{2}{|l|}{ Number of sex partners } \\
\hline-1 & $58(95.1)$ \\
\hline$-\geq 1$ & $3(4.9)$ \\
\hline \multicolumn{2}{|l|}{ Number of UTI in the past 6 months } \\
\hline-2 & $19(31.2)$ \\
\hline$-\geq 2$ & $42(68.8)$ \\
\hline Median number of UTI in the past 6 months & 5 (IQR 4-8) \\
\hline Median number of antibiotic treatment cycle in the year before & $6($ IQR 3-10) \\
\hline \multicolumn{2}{|l|}{ Hormonal status } \\
\hline - Premenopausal & $40(65.5)$ \\
\hline - Postmenopausal & $21(34.5)$ \\
\hline \multicolumn{2}{|l|}{ Isolated strains before antibiotic treatment } \\
\hline - Escherichia coli & $42(68.8)$ \\
\hline - Klebsiella spp. & $8(13.1)$ \\
\hline - Enterococcus spp. & $7(11.5)$ \\
\hline - Others & $4(6.5)$ \\
\hline
\end{tabular}

Statistical analysis

As null hypothesis, we consider that there is no difference in terms of QoL between baseline and the third follow- up evaluation. Moreover, our null hypothesis is that there is no difference in terms of number of symptomatic recurrences between the follow-up period and 1 year before enrolment. In order to obtain significant results to analyze, sample size calculation was based on the following assumptions: difference in terms of recurrence between baseline and follow-up visit: $-3 \pm 1$; $\alpha$ error level, 0.05 two-sided; statistical power, $80 \%$; anticipated effect size, Cohens $d=0.5$. The calculation yielded 43 individuals. Taking into account a drop-out rate of $10 \%$, the final sample size was set to 49 patients. The statistical analysis was performed as follows: continuous variables are presented as median and InterQuartile Range (IQR) and categorical variables are presented as absolute (n) and relative (\%) frequency distributions. $t$-tests were used to compare average performance between enrolment and the follow-up evaluation, and between the periods before and after enrolment. The statistical analysis was performed using SPSS.

\section{Results}

A total of 67 patients were screened in this study, 61 of whom were finally included and treated, with a median age of 41.3 (ranging from 23 to 53) years. Six patients declined to be enrolled for nonclinical related reasons.

\section{Enrolment time}

All demographic and baseline clinical and microbiological characteristics are summarized in Table 1 . The most common isolated strain was Escherichia coli $(42 / 61 ; 68.8 \%)$, followed by Klebsiella and Enterococcus spp. Absence of infection was reported in all women after the antibiotic treatments before enrolment. The median number of UTI per 6 months, in the year before, was 5 (IQR 4-8). The median number of antibiotic treatment cycles was six in the year before (IQR 3-10). 


\begin{tabular}{|c|c|c|c|c|c|}
\hline Outcomes variable & Baseline & Follow-up 30 days & Follow-up 90 days & Follow-up 180 days & p-value \\
\hline \multicolumn{6}{|l|}{ Clinical improvement } \\
\hline Symptoms related to UTI and antibiotic use & $0 / 61$ & $1 / 61(1.6)$ & $2 / 61(3.4)$ & $4 / 61(6.5)$ & $<0.001^{\dagger}$ \\
\hline Moderate improvement without antibiotic use & - & 19/61 (31.2) & 12/61 (19.6) & $6 / 61(9.8)$ & \\
\hline Restore to pre-UTI situation & - & $41 / 61(67.2)$ & $47 / 61(77.0)$ & $51 / 61(83.6)$ & \\
\hline QoL (questionnaire) & 94.2 & 98.6 & 98.7 & 99.1 & $<0.001^{\dagger}$ \\
\hline \multicolumn{6}{|l|}{ Microbiological improvement } \\
\hline Bacterial growth (before $A B T$ treatment) & $61 / 61$ & $1 / 61(1.6)$ & $2 / 61(3.4)$ & $4 / 61(6.5)$ & $<0.001^{\dagger}$ \\
\hline \multicolumn{6}{|l|}{ Isolated strains } \\
\hline - Escherichia coli & $42(68.8)$ & $1(1.6)$ & $1(1.6)$ & $3(4.9)$ & \\
\hline - Klebsiella spp. & $8(13.1)$ & & $1(1.6)$ & & \\
\hline - Enterococcus spp. & $7(11.5)$ & & & & \\
\hline - Proteus spp. & $2(3.3)$ & & & $1(1.6)$ & \\
\hline - Serratia spp. & $2(3.3)$ & & & & \\
\hline Transition to ABU from UTI & - & - & $11 / 61(18.1)$ & $18 / 61(29.5)$ & \\
\hline \multicolumn{6}{|l|}{ Isolated strains } \\
\hline - Enterococcus spp. & - & - & $11 / 61(18.1)$ & $18 / 61(29.5)$ & \\
\hline Antibiotic treatment cycle (median) & $6($ IQR 3-10) & & & $2($ IQR $0-3)$ & $<0.001^{\ddagger}$ \\
\hline \multicolumn{6}{|c|}{$\begin{array}{l}{ }^{\dagger} \text { Difference between baseline and each follow-up visits. } \\
{ }_{\text {† }} \text { Difference between the period before and after enrolment in terms of antibiotic consumption. } \\
\text { IQR: Interquartile range; QoL: Quality of life. }\end{array}$} \\
\hline
\end{tabular}

\section{Clinical \& microbiological data at the first follow-up evaluation (30 days)}

All women reported high adherence to the treatment $(61 / 61 ; 100 \%)$. A total of 41 out of 61 enrolled women (67.2\%) reported an improvement of QoL (QoL 94.2 vs 98.6; $p$ < 0.001) and return to pre-UTI clinical status. A total of 19 patients (31.2\%) reported a mild, but not statistically significant improvement of QoL, that, however, not required antibiotic use. Only one patient showed a symptomatic episode that required antibiotic treatment $(1.6 \%)$. No significant AEs were reported. All microbiological data are shown in Table2.

\section{Clinical \& microbiological data at the second follow-up evaluation (3 months)}

At the second follow-up, 47/61 (77\%) reported a significant improvement of QoL (QoL 94.1 vs 98.7; p < 0.001 ), while 12 a mild, but not statistically significant improvement of QoL, that, however, not required antibiotic use (19.6\%). Two women reported a symptomatic episode that required antibiotic treatment for symptomatic UTI (3.4\%). From a microbiological point of view, in 11 patients out of 61 (18.1\%), we observed a transition from symptomatic UTI to asymptomatic bacteriuria (ABU) (from E. coli to Enterococcus faecalis). No AEs were reported. All microbiological data are shown in Table 2.

\section{Clinical \& microbiological data at the last follow-up evaluation (6 months)}

No missing administrations were reported, with $100 \%$ adherence. No clinical relevant AEs were reported during the whole study period. At the last follow-up examination, 51/61 (83.6\%) reported an improvement of QoL $(\mathrm{QoL}$

94.2 vs $99.1 ; p<0.001)$ and return to their clinical status before UTI. Six patients reported a mild, but not statistically significant improvement of QoL, that, however, not required antibiotic use $(9.8 \%)$. On the other hand, 4/61 women reported at least one symptomatic episode that required antibiotic treatment for symptomatic UTI $(6.5 \%)$. In the period after enrolment, we found a statistically significant difference in terms of number of antibiotic treatment cycles, when compared with the previous period (median 6 [IQR $3-$ 10] vs 2 [IQR 0-3]; p < 0.0001). Finally, in 18 patients out of 61 (29.5\%), we observed a transition from symptomatic UTI to ABU (from E. coli to

E. faecalis) (Table 2).

Treatment failure

\section{Subanalysis of patients who required antibiotic treatment}

In the whole study period, seven women reported at least one symptomatic UTI recurrence that required antibiotic treatment (11.4\%). Two patients required two antibiotic treatment cycles. In these patients, the most common 


\begin{tabular}{|c|c|c|c|}
\hline Clinical and microbiological data & Who required $\mathrm{ABT}$ & Who did not required $A B T$ & p-value \\
\hline No. of total patients & 7 & 54 & - \\
\hline Median age (range) & $41.5(27-53)$ & $41.2(23-51)$ & 1.0 \\
\hline \multicolumn{4}{|l|}{ Educational level } \\
\hline - Primary school & - & - & 0.22 \\
\hline - Secondary school & $3(42.8)$ & $17(31.5)$ & \\
\hline Postsecondary education & $4(57.2)$ & $37(68.5)$ & \\
\hline \multicolumn{4}{|l|}{ Current smoker } \\
\hline - No & $2(28.6)$ & $17(31.5)$ & 1.0 \\
\hline - Yes & $5(71.4)$ & $37(68.5)$ & \\
\hline Sexually active (past month) & $6(85.7)$ & $53(98.1)$ & 0.21 \\
\hline \multicolumn{4}{|l|}{ Number of sex partners } \\
\hline-1 & $6(85.7)$ & $52(96.3)$ & 0.31 \\
\hline$-\geq 1$ & $1(14.3)$ & $2(3.7)$ & \\
\hline \multicolumn{4}{|l|}{ Number of UTI in the past 6 months } \\
\hline-2 & $2(28.6)$ & $17(31.5)$ & 1.0 \\
\hline$-\geq 2$ & $5(71.4)$ & $37(68.5)$ & \\
\hline Median number of UTI in the past 6 months & 5 (IQR 4-8) & $4(\mathrm{IQR} 4-6)$ & 0.43 \\
\hline Median number of antibiotic treatment cycle in the year before & $6($ IQR 3-10) & $4(\mathrm{IQR} 3-5)$ & 0.09 \\
\hline \multicolumn{4}{|l|}{ Hormonal status } \\
\hline - Premenopausal & $3(42.8)$ & $37(68.5)$ & 0.22 \\
\hline - Postmenopausal & $4(57.2)$ & $17(31.5)$ & \\
\hline \multicolumn{4}{|l|}{ Isolated strains before $A B T$ treatment } \\
\hline - E. coli & $6(85.7)$ & $36(66.7)$ & 0.41 \\
\hline - Klebsiella spp. & $1(14.3)$ & $7(12.9)$ & \\
\hline - Enterococcus spp. & & $7(12.9)$ & \\
\hline - Others & & $4(7.5)$ & \\
\hline \multicolumn{4}{|l|}{ Isolated strains after enrolment } \\
\hline - Klebsiella spp. & $5(71.4)$ & - & - \\
\hline - Escherichia coli & $2(28.6)$ & - & \\
\hline
\end{tabular}

isolated strain was Klebsiella pneumoniae (71.4\%) (Table 3). From a clinical point of view, no statistically significant difference was found in terms of demographic or clinical characteristics when compared with patients who did not required antibiotic treatment (Table 3). The only clinical characteristic that seems different from the other group of patients, is the previous use of antibiotics and the number of previous UTI, even if it is not statistically significant.

\section{Discussion}

Although antibiotics are still considered the pillar for the management of UTIs, the spread of multidrugresistant microorganisms is worryingly rising and requires thorough monitoring and novel preventive approaches. Indeed, improper antibiotic abuse and antimicrobial resistance have become significant worldwide issues. As a result, in the last few years interest and research in the nonantibiotic prophylaxis of rUTIs have gradually grown. In particular, the use of phytotherapy and nutraceuticals might represent a feasible and effective, alternative approach to curtail the use of antibiotics and the rate of symptomatic recurrences $[3,13,19,20]$. Recently, Cai et al. published a paper about the efficacy of a combination of Hibiscus extract, vegetable proteases and Commiphora myrrha extract, for preventing symptomatic UTI episodes and improving patients $\mathrm{QoL}_{[12]}$.

Here, we report the first prospective observational study evaluating the efficacy and prophylactic effect of an oral supplementation of xyloglucan, hibiscus and propolis in women suffering from recurrent uncomplicated UTIs. In our experience, this medical device was adequately tolerated, resulting in optimal compliance as no side effects were registered. Moreover, it showed remarkable results since almost $84 \%$ of patients reported a notable clinical improvement of QoL with a statistically significant difference compared with baseline after 6 months from the 
beginning of the treatment. Noteworthy, from a microbiological point of view, we observed a transition from symptomatic UTI to ABU in nearly a third of the entire cohort. Several authors recently argued that ABU could play a protective role in preventing symptomatic recurrences, inhibiting the colonization of enteric pathogens, such as E. coli. The term ABU refers to isolation of bacteria in an appropriately collected urine specimen from an individual without symptoms of urinary tract infection who do not require antibiotic treatment. In this sense, ABU is not a recurrence. Indeed, recent data have begun to unravel patient and pathogen-related factors influencing rUTI, suggesting that its management will likely require considering also bacterial reservoirs at anatomical sites other than the urinary tract. In a randomized clinical trial conducted by Cai et al. [13], after a 1-year period of antimicrobial therapy for ABU in women affected by recurrent UTIs, the rate of symptomatic UTIs was even higher in those treated with antibiotics. In this sense, Cai et al. demonstrated, in women affected by recurrent UTI, that ABU due to E. faecalis seems an important defense mechanism that effectively interferes with the establishment of many important enteric pathogens, such as E. coli, reducing the symptomatic recurrences. In this light, the use of an antibiotic-sparing approach should be preferred since it does not alter the typical intestinal microbiota. In this regard, the fact that this medical device was able to establish an ABU is solid proof of the absence of its effects on normal microbiota. Xyloglucan is a plant polymer extracted from the seeds of the tamarind tree (Tamarindus indica) and is usually described as a mucosal protector', as it has been shown to be able to produce a protective film over the mucous membranes on human epithelial tissues [21]. Additional in vitro studies have demonstrated that xyloglucan can reduce the intestinal adhesion and reservoir of uropathogenic $E$. coli strains $[8,10]$, and by interfering in the process by which microorganisms contact uroepithelial cell receptors [20]. On the other hand, $H$. sabdariffa is a phytocomplex containing sambubiosides, which seems to have an antiinflammatory effect by reducing the levels of inflammatory mediators such as iNOS, NO, IL-6, MCP-1 and TNF- $\alpha$ induced by lipopolysaccharide (LPS), in both cell and animal models ${ }_{[22]}$. Moreover, in previous studies, H. sabdariffa has been shown to have anti- adhesive properties at a urinary level, as it is capable of inhibiting biofilm creation by common community-acquired isolates ${ }_{[23]}$. Propolis is a resinous substance obtained by bees from exudates and buds of plants. In a double-blind, randomized, clinical trial, the propolis excreted in urine also had a significant bacterial anti-adhesive effect, thus supporting the use of propolis to prevent recurrent UTIs [24]. Since propolis and hibiscus are systemically absorbed, they can play this protective role directly at a urinary level, thus preventing the adhesion of uropathogenic bacteria. Conversely, in the case of xyloglucan, its protective effect is likely to be mainly exerted on the intestinal tract, since it is not absorbed. Our results confirm the capability of the components of this medical device to reduce the rate of symptomatic UTI recurrences and the use of antimicrobial therapies. Indeed, in our experience, a statistically significant difference in terms of median number of antibiotic treatment cycles was found between the study period and the period before enrollment (mean 6 vs $2 ; p<0.0001$ ). Ultimately, the lack of a gold-standard treatment for the management of rUTIs and the risk of increasing resistance among microorganisms are two important reasons why we need to seek novel nonantibiotic approaches. Based on these preliminary results, further clinical studies should be performed to assess the preventive effect of this medical device. Indeed, larger high-quality studies will be required to confirm these promising findings. However, only advancing our understandings about the effect of phytotherapy and nutraceuticals will be able to develop appropriate nonantibiotic treatment and prevention strategies for rUTIs.

\section{Conclusion}

In conclusion, the medical device containing xyloglucan, hibiscus and propolis seems to be able to improve quality of life in women affected by rUTIs, reducing symptomatic episodes and the use of antibiotics. In this sense, this antibiotic-sparing approach to women affected by recurrent UTIs seems an interesting tool for use in everyday clinical practice for improving antimicrobial stewardship.

\section{Financial \& competing interests disclosure}

The authors have no relevant affiliations or financial involvement with any organization or entity with a financial interest in or financial conflict with the subject matter or materials discussed in the manuscript. This includes employment, consultancies, honoraria, stock ownership or options, expert testimony, grants or patents received or pending, or royalties.

No writing assistance was utilized in the production of this manuscript. 
- Alarming increase in antimicrobial resistance has stimulated interest in an antibiotic-sparing approach for the management of urinary tract infections.

- An oral medical device containing xyloglucan (a natural hemicellulose), propolis and hibiscus has been recently introduced in the international pharmacopoeia for the prevention of urinary tract infections.

- This clinical trial evaluated the efficacy of a medical device containing xyloglucan, hibiscus and propolis in the management of women affected by recurrent urinary tract infections.

- All patients received one capsule a day for 15 days (one cycle every month, for 6 months).

- Xyloglucan, propolis and hibiscus treatment is able to improve patients' quality of life and restore pre-UTI clinical status.

- This medical device, containing xyloglucan, propolis and hibiscus, is able to reduce antibiotic use in women with recurrent UTIs.

- Xyloglucan, propolis and hibiscus treatment seems an interesting antibiotic-sparing approach in women affected by recurrent UTIs.

\section{References}

Papers of special note have been highlighted as: • of interest; $\bullet \bullet$ of considerable interest

1. Medina M, Castillo-Pino E. An introduction to the epidemiology and burden of urinary tract infections. Ther. Adv. Urol. 11, 1756287219832172 (2019).

2. Storme O, TiránSaucedo J, Garcia-Mora A et al. Risk factors and predisposing conditions for urinary tract infection. Ther. Adv. Urol.11, 1756287218814382 (2019).

3. Cai T, Cocci A, Tiscione D et al. L-Methionine associated with Hibiscus sabdariffa and Boswellia serrata extracts are not inferior to antibiotic treatment for symptoms relief in patients affected by recurrent uncomplicated urinary tract infections: focus on antibiotic-sparing approach. Arch. Ital. Urol. Androl. 90(2), 97-100(2018).

.- Shows the utility of the antibiotic-sparing approach in the management of urinary tract infections.

4. Rich SN, Klann EM, Almond CR et al. Associations between antibiotic prescriptions and recurrent urinary tract infections in female college students. Epidemiol. Infect. 147, e119 (2019).

5. Naber KG, Bergman B, Bishop MC et al. Urinary Tract Infection (UTI) Working Group of the Health Care Office (HCO) of the European Association of Urology (EAU). EAU guidelines for the management of urinary and male genital tract infections. Eur. Urol. 40, 576-588 (2001).

6. Bonkat G, Bartoletti R, Cai T et al. EAU guidelines on urological infections (2019). https://uroweb.org/guideline/urologicalinfections

7. Costache RC, Novac B, Bardan TR et al. Xyloglucan + gelose combination versus placebo as adjuvant therapy to first-line antimicrobials for uncomplicated urinary tract infection in adults. Urol. Int. 19, 1-8(2019).

- Shows the utility of the medical device containing xyloglucan, hibiscus and propolis for improving urinary tract infection symptoms and patients' quality of life.

8. De Servi B, Ranzini F, Piqué N. Effect of Utipro $\left({ }^{\mathcal{S}}\right)$ (containing gelatin-xyloglucan) against Escherichia coli invasion of intestinal epithelial cells: results of an in vitro study. Fut. Microbiol. 11(5), 651-658 (2016).

- In vitro study showing the protective effect of xyloglucan against Escherichia coli invasion of intestinal cells, reducing the settling of $E$. coli reservoirs.

9. Olier M, Sekkal S, Harkat C et al. Evaluation of reticulated gelatin-hibiscus-propolis against intestinal commensal species commonly associated with urinary tract infections. Fut. Microbiol. 12(6), 505-513(2017).

10. Fraile B, Alcover J, Royuela M et al. Xyloglucan, hibiscus and propolis for the prevention of urinary tract infections: results of in vitro studies. Fut. Microbiol. 12(8), 721-731 (2017).

- In vitro study showing the protective role of xyloglucan in the prevention of urinary tract infection.

11. Chakalova G. Utipro plus capsule, a new innovative approach for the treatment of cystitis and other bacterial urinary tract infections.

Akush Ginekol (Sofiia). 55(Suppl. 1 Pt 2), 23-25 (2016).

12. Cai $\mathrm{T}$, Tiscione $\mathrm{D}$, Cocci A et al. Hibiscus extract, vegetable proteases and Commiphora myrrha are useful to prevent symptomatic UTI episode in patients affected by recurrent uncomplicated urinary tract infections. Arch. Ital. Urol. Androl. 90(3), 203-207 (2018).

- Interesting clinical trial demonstrating the utility of hibiscus to prevent symptomatic episodes of recurrent urinary tract infection.

13. Cai T, Nesi G, Mazzoli S et al. Asymptomatic bacteriuria treatment is associated with a higher prevalence of antibiotic resistant strains in women with urinary tract infections. Clin. Infect. Dis. 61(11), 1655-1661 (2015).

14. Hooton TM, Scholes D, Gupta K et al. Amoxicillin-clavulanate vs ciprofloxacin for the treatment of uncomplicated cystitis in women: a randomized trial. JAMA 293, 949-955 (2005). 
15. Mazzoli S, Cai T, Rupealta V et al. Interleukin 8 and anti-Chlamydia trachomatis mucosal IgA as urogenital immunologic markers in patients with C. trachomatis prostatic infection. Eur. Urol. 51, 1385-1393 (2007).

16. Cai T, Tamanini I, Kulchavenya $\mathrm{E}$ et al. The role of nutraceuticals and phytotherapy in the management of urinary tract infections: what we need to know? Arch. Ital. Urol. Androl. 89(1), 1-6 (2017).

17. Kaplan RM, Bush JW, Berry CC. Health status: types of validity and the index of wellbeing. Health Serv. Res. 11, 478-507 (1976).

18. Ledda A, Belcaro G, Dugall $\mathrm{M}$ et al. Highly standardized cranberry extract supplementation (Anthocran ${ }^{\mathcal{S}}$ ) as prophylaxis in young healthy subjects with recurrent urinary tract infections. Eur. Rev. Med. Pharmacol. Sci. 21(2), 389-393 (2017).

19. Stange R, Schneider B, Albrecht U et al. Results of a randomized, prospective, double-dummy, double-blind trial to compare efficacy and safety of a herbal combination containing Tropaeoli majoris herba and Armoraciae rusticanae radix with cotrimoxazole in patients with acute and uncomplicated cystitis. Res. Rep. Urol. 9, 43-50(2017).

20. Beerepoot M, Geerlings S. Non-antibiotic prophylaxis for urinary tract infections. Pathogens 5(2), pii:E36 (2016).

21. Piqué N, Gómez-Guillén MDC, Montero MP. Xyloglucan, a plant polymer with barrier protective properties over the mucous membranes: an overview. Int. J. Mol. Sci. 19(3), 2018.

22. Sogo T, Terahara N, Hisanaga A et al. Anti-inflammatory activity and molecular mechanism of delphinidin 3-sambubioside, a Hibiscus anthocyanin. Biofactors 41(1), 58-65 (2015).

23. Alshami I, Alharbi AE. Hibiscus sabdariffa extract inhibits in vitro biofilm formation capacity of Candida albicans isolated from recurrent urinary tract infections. Asian Pac. J. Trop. Biomed. 4(2), 104-108 (2014).

24. Lavigne JP, Vitrac X, Bernard L et al. Propolis can potentialise the anti-adhesion activity of proanthocyanidins on uropathogenic Escherichia coliin the prevention of recurrent urinary tract infections. BMC Res. Notes 4, 522 (2011). 